\title{
OPEN Observational study on wearable biosensors and machine learning-based remote monitoring of COVID-19 patients
}

Ka-Chun Un ${ }^{1,5}$, Chun-Ka Wong ${ }^{1,5}$, Yuk-Ming Lau ${ }^{1}$, Jeffrey Chun-Yin Lee ${ }^{1}$, Frankie Chor-Cheung Tam ${ }^{1}$, Wing-Hon Lai ${ }^{1}$, Yee-Man Lau ${ }^{1}$, Hao Chen ${ }^{2}$, Sandi Wibowo ${ }^{2}$, Xiaozhu Zhang ${ }^{2}$, Minghao Yan ${ }^{2}$, Esther Wu ${ }^{2}$, Soon-Chee Chan², Sze-Ming Lee ${ }^{3}$, Augustine $\mathrm{Chow}^{3}$, Raymond Cheuk-Fung Tong ${ }^{3}$, Maulik D. Majmudar ${ }^{2}$, Kuldeep Singh Rajput ${ }^{2}$, Ivan Fan-Ngai Hung ${ }^{4} \&$ Chung-Wah Siu ${ }^{1 \bowtie}$

Patients infected with SARS-CoV-2 may deteriorate rapidly and therefore continuous monitoring is necessary. We conducted an observational study involving patients with mild COVID-19 to explore the potentials of wearable biosensors and machine learning-based analysis of physiology parameters to detect clinical deterioration. Thirty-four patients (median age: 32 years; male: $52.9 \%$ ) with mild COVID-19 from Queen Mary Hospital were recruited. The mean National Early Warning Score 2 (NEWS2) were $0.59 \pm 0.7 .1231$ manual measurement of physiology parameters were performed during hospital stay (median 15 days). Physiology parameters obtained from wearable biosensors correlated well with manual measurement including pulse rate $(r=0.96, p<0.0001)$ and oxygen saturation $(r=0.87, p<0.0001)$. A machine learning-derived index reflecting overall health status, Biovitals Index (BI), was generated by autonomous analysis of physiology parameters, symptoms, and other medical data. Daily BI was linearly associated with respiratory tract viral load $(p<0.0001)$ and NEWS2 $(r=0.75$, $p<0.001$ ). BI was superior to NEWS2 in predicting clinical worsening events (sensitivity $94.1 \%$ and specificity $88.9 \%$ ) and prolonged hospitalization (sensitivity $66.7 \%$ and specificity $72.7 \%$ ). Wearable biosensors coupled with machine learning-derived health index allowed automated detection of clinical deterioration.

Coronavirus Disease 2019 (COVID-19) due to severe acute respiratory syndrome coronavirus-2 (SARS-CoV-2) has spread at an alarming rate and resulted in a pandemic causing more than 12 million infections and 560,000 deaths globally as of July $122020^{1-4}$. The overwhelmingly large number of COVID-19 patients, together with the shortage of hospital beds and isolation facilities beleaguered healthcare systems worldwide ${ }^{5-9}$. Despite having initially mild symptoms, COVID-19 patients may still deteriorate during hospital stay; for instance, onethird of COVID-19 patients with mild or moderate disease on admission deteriorated and required mechanical ventilation in intensive care units ${ }^{1,3,10,11}$. Thus, early recognition of patient deterioration is crucial for successful clinical management. Conventionally, hospitalized patients are assessed intermittently by manual measurement of physiology parameters and early warning score systems such as National Early Warning System 2 (NEWS2) are used to identify deteriorating patients (Appendix Table 2$)^{12-14}$. Amidst the pandemic, manual measurement of physiology parameters by healthcare workers inevitably increases their exposure to the virus and risk of contracting COVID-19, especially with the constrained personal protective equipment (PPE) supply ${ }^{15,16}$. In fact, as of April 8 2020, 22,073 healthcare workers from 52 countries were infected by COVID-19 according to situation report issued by the World Health Organization (WHO $)^{17}$.

Biosensors for physiology parameters have been progressively miniaturized in recent years to allow incorporation into wearable devices such as armbands and wristbands, enabling monitoring of heart rate, heart rate variability, respiration rate, oxygen saturation, blood pulse wave, skin temperature and actigraphy in a continuous

${ }^{1}$ Cardiology Division, Department of Medicine, The University of Hong Kong, Hong Kong SAR, China. ${ }^{2}$ Biofourmis Singapore Pte. Ltd, Singapore, Singapore. ${ }^{3}$ Harmony Medical Inc, Hong Kong SAR, China. ${ }^{4}$ Infectious Diseases Division, Department of Medicine, The University of Hong Kong, Hong Kong SAR, China. ${ }^{5}$ These authors contributed equally: Ka-Chun Un and Chun-Ka Wong. ${ }^{凶}$ email: cwdsiu@hku.hk 
and autonomous manner ${ }^{18-23}$. As state-of-the-art telecommunication technologies allow instantaneous and multidirectional massive data transfer at low cost, it is now possible to remotely monitor a large number of patients in a real-time ${ }^{24}$, and to relay information to clinicians for timely intervention. Conceivably, in the setting of COVID-19, remote patient monitoring tools enable healthcare workers to manage infected patients with minimal physical contact, thereby minimizing their risk of getting infected, as well as reducing the consumption of PPE. Furthermore, machine learning-based analytic systems may enhance clinical management by detecting early signs of clinical deterioration from continuous streams of physiology data collected from wearable biosensors, allowing prompt and precise delivery of intervention, and optimization of resources use. In this study, we explored the potential of utilizing wearable biosensors and machine learning-based remote monitoring platform for managing COVID-19 patients hospitalized in isolation wards.

\section{Methods}

Study design and participants. This observational study was conducted in the Department of Medicine, Queen Mary Hospital, The University of Hong Kong, Hong Kong. The study aimed to evaluate the feasibility of utilizing wearable biosensors and machine learning-based analysis of multivariate longitudinal physiology parameters in the management of COVID-19 patients. Patients hospitalized with PCR confirmed COVID-19 were recruited. Patients were excluded if they were under 18 years of age, had NEWS $2>4$ on admission, required intensive care on admission, or lacked skills in operating simple electronic devices. In addition to the standard management, study participants were continuously monitored using wearable biosensors and machine learning-based remote monitoring platform during hospital stay. Written informed consent were obtained from all recruited participants. The study protocol was approved by the institutional review board of the University of Hong Kong/Hospital Authority Hong Kong West Cluster and registered in the clinicaltirals.gov (NCT04343794). This clinical trial protocol follows the Standard Protocol Items: Recommendations for Interventional Trials $\left(\right.$ SPIRIT) ${ }^{25,26}$. The underlying protocol follows the Consolidated Standards of Reporting Trials (CONSORT) ${ }^{27,28}$.

Isolation ward setting and routine monitoring. All COVID-19 patients were initially managed in isolation wards and were transferred to intensive care unit or stepdown wards when necessary. Depending on the initial clinical condition, COVID-19 patients in isolation wards had their physiology parameters monitored every 4-hourly to 12 -hourly by healthcare workers wearing recommended PPE.

Wearable biosensors-based remote monitoring platform. Patients were remotely monitored using Biovitals Sentinel Platform (Biofourmis, Boston, MA, USA), which consisted of wearable biosensors, patientfacing smartphone application, secured cloud for data hosting and processing, and web-based dashboard for clinicians. (Fig. 1) The wearable biosensor, Everion (Biofourmis, Boston, MA, USA), was a continuous physiology parameters monitoring device worn on the upper arm to capture multi-dimensional physiology parameters including heart rate, heart rate variability, respiration rate, oxygen saturation, blood pulse wave, skin temperature and actigraphy. Patients were instructed to wear the wearable biosensors for $23 \mathrm{~h}$ per day and charge them while showering. The wearable biosensors continuously transmitted physiology data via Bluetooth to paired smartphones. In addition, patients were instructed to report their symptoms using the dedicated smartphone application. All data were automatically transferred in real-time to a secured cloud for hosting and analysis via cellular network. Physiology data, patient-reported symptoms and machine-learning derived analytic results were displayed on a web-based dashboard at the nursing station and the clinicians' office for healthcare workers to review.

Machine learning for COVID-19 progression monitoring. The cloud based analytical system used, Biovitals Analytics Engine (Biofourmis, Boston, MA, USA), was a machine learning system developed as a disease-specific predictive management tool which received $510(\mathrm{k})$ clearance from the U.S. Food and Drug Administration as a medical device for ambulatory physiology monitoring ${ }^{29}$. One of its output, the Biovitals Index, was a machine learning-derived health index that reflected the overall health status of users. It was generated by autonomous analysis of physiology parameters, symptoms, and other medical data ${ }^{29}$ (Fig. 1). Higher Biovitals Index reflected worse health status and a longer duration of further hospital stay is expected, and vice versa. The machine learning and statistical methods for derivation of Biovitals Index were described in previous publication $^{29}$, Supplementary Appendix and Appendix Table 1.

Measures and outcomes. The primary virologic outcome was the viral load from respiratory sample estimated semi-quantitatively using RT-PCR cycle threshold (Ct) value for SARS-CoV-2, which was sampled every 2-4 days in the isolation wards after admission. The viral load was classified as "high" with Ct value $\leq 20$, "medium" with Ct value 20-30, "low" with Ct value $>30$, and "undetected". The clinical status was assessed on admission and every morning during the hospital stay using NEWS2, which is endorsed by the British National Health System for initial assessment and serial monitoring of patients with sepsis to early detect clinical deterioration $^{30}$ (Appendix Table S2). Clinical worsening event was defined as the new occurrence of one or more of the followings: (1) NEWS2 $\geq 5$, (2) individual component parameter constituting NEWS2 $=3$, (3) increase in daily NEW2 $\geq 3$, (4) use of oxygen therapy, and (5) intensive care unit admission. COVID-19 patients were discharged from the hospital after fulfilling the current discharge criteria including (1) clinical criteria, that is when the clinical conditions of the patients improve and afebrile for at least $24 \mathrm{~h}$ and (2) laboratory evidence of SARS$\mathrm{CoV}-2$ clearance as evidenced by negative RT-PCR tests for nasopharyngeal swab with sampling interval $\geq 24 \mathrm{~h}$. 
A

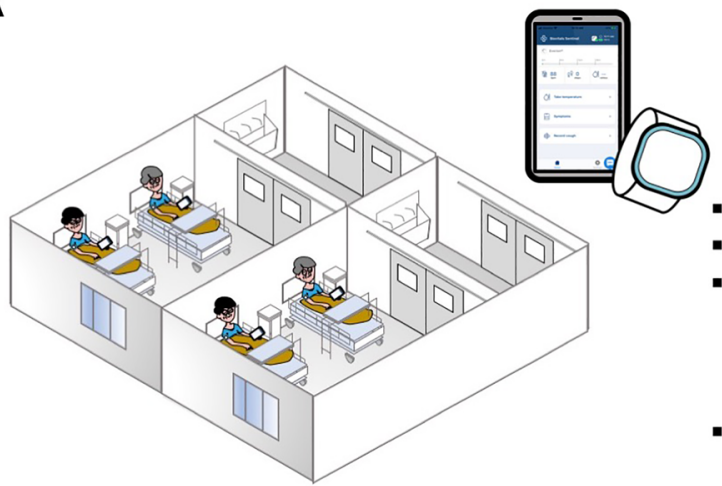

\section{Continuous Physiology \\ Parameters Monitoring in Isolation Ward}

- Wearable multi-parameter biosensor

- 24/7 fully-automated data collection

- Heart rate, heart rate variability, respiration rate, oxygen saturation, blood pulse wave, skin temperature and actigraphy

- Dedicated patient-facing smartphone application

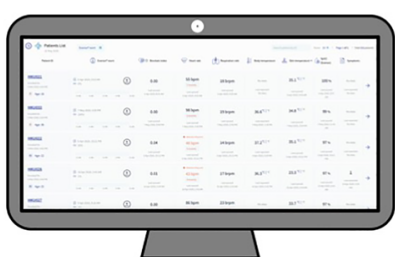

\section{Clinician Dashboard (Nursing Station)}

\section{Secured Cloud Storage and Analytic Platform}

- Available on computers and smartphones

- Real-time display of physiology data

- Alert clinicians on abnormal results

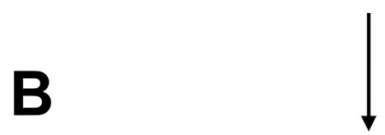

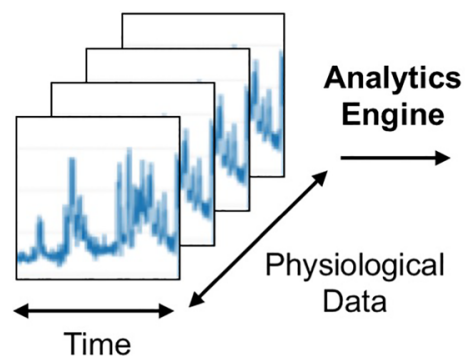

Time

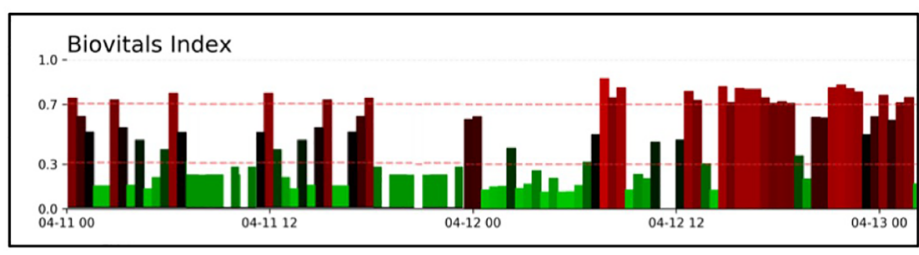

Machine learning-derived Biovitals Index was generated for each patient with the physiology data collected

Figure 1. (A) Isolation ward setting and study design. (B) Machine learning-based derivation of Biovitals Index with continuous streams of physiology data from wearable biosensors.

Statistical analysis. Continuous and discrete variables were expressed as mean \pm standard deviation and percentages, respectively. Chi-square test or Fisher's exact test was used to compare categorical variables between groups. Student's $t$ test or Mann-Whitney $U$ test was performed to compare continuous variables. Pearson correlation test was used for evaluating the linear association between two variables. Analysis of variance (ANOVA test) was used to compare the sample mean between multiple groups. Receiver operating characteristics (ROC) curve was used for evaluating the trade-off between sensitivity and specificity. Area under the curve (AUC) of ROC was used for evaluating the predictive accuracy for classification models. All tests were two-sided, and a $p$-value $<0.05$ was considered significant. All statistical analyses were performed using SPSS software (version 21.0), R (version 3.6.1), and Python (version 3.6) programming languages. 


\begin{tabular}{|c|c|}
\hline Clinical characteristics & $(n=34)$ \\
\hline \multicolumn{2}{|l|}{ Demographics and co-morbidities } \\
\hline Age, years & $32(21-50)$ \\
\hline Male, n (\%) & $18(52.9)$ \\
\hline Hypertension, $\mathrm{n}(\%)$ & $2(5.9)$ \\
\hline Diabetes mellitus, $\mathrm{n}(\%)$ & $3(8.8)$ \\
\hline Stroke, $\mathrm{n}(\%)$ & $1(2.9)$ \\
\hline \multicolumn{2}{|l|}{ Symptoms } \\
\hline Fever, n (\%) & $19(55.9)$ \\
\hline Cough, n (\%) & $13(38.2)$ \\
\hline Sore throat, n (\%) & $7(22.2)$ \\
\hline Sputum, n (\%) & $6(17.6)$ \\
\hline Shortness of breath, $\mathrm{n}(\%)$ & $2(5.9)$ \\
\hline Vomiting, n (\%) & $1(2.9)$ \\
\hline Diarrhea, n (\%) & $11(11.3)$ \\
\hline Fatigue, n (\%) & $3(8.8)$ \\
\hline Myalgia, n (\%) & $3(8.8)$ \\
\hline Loss of taste and/or smell, n (\%) & $8(23.5)$ \\
\hline \multicolumn{2}{|l|}{ Vital measurement } \\
\hline Systolic blood pressure, $\mathrm{mmHg}$ & $138(121-153)$ \\
\hline Diastolic blood pressure, $\mathrm{mmHg}$ & $85(77-92)$ \\
\hline Heart rate, bpm & $90(81-99)$ \\
\hline $\mathrm{SpO}_{2}, \%$ & $98(97-99)$ \\
\hline NEWS2 score & $0.59 \pm 0.7$ \\
\hline \multicolumn{2}{|l|}{ Chest radiographic findings } \\
\hline Normal & $8(23.5)$ \\
\hline Unilateral infiltrates & $15(44.1)$ \\
\hline Bilateral infiltrates & $11(32.4)$ \\
\hline \multicolumn{2}{|l|}{ Laboratory findings } \\
\hline White cell counts, $1 \times 10^{9} / \mathrm{ml}$ & $6.4 \pm 2.2$ \\
\hline Lymphocyte counts, $1 \times 10^{9} / \mathrm{ml}$ & $1.6 \pm 0.6$ \\
\hline Hemoglobin, $\mathrm{g} / \mathrm{dl}$ & $14.3 \pm 1.8$ \\
\hline Platelet counts, $1 \times 10^{12} / \mathrm{ml}$ & $257 \pm 69$ \\
\hline Alanine aminotransferase $\mathrm{U} / \mathrm{l}$ & $30.8 \pm 23.1$ \\
\hline Creatinine, $\mu \mathrm{mol} / \mathrm{L}$ & $77.8 \pm 14.5$ \\
\hline Elevated troponin level, n (\%) & $1(2.9)$ \\
\hline
\end{tabular}

Table 1. Clinical characteristics of the study population.

\section{Results}

Between March 19, 2020 and April 11, 2020, 37 patients with PCR-confirmed COVID-19 were admitted to the isolation wards of Queen Mary Hospital. 34 patients fulfilling the inclusion and exclusion criteria were recruited. The median age was 32 years (IQR 21-50 years), and 52.9\% were men. Table 1 summarized the clinical characteristics of the study population. On admission, 19 (55.9\%) patients presented with fever, and 13 (38.2\%) had cough. The median systolic and diastolic blood pressure were $138 \mathrm{mmHg}(121-153 \mathrm{mmHg})$ and $85 \mathrm{mmHg}$ (77-92 $\mathrm{mmHg}$ ) respectively, and the median heart rate was $90 \mathrm{bpm}$ (81-99 bmp). No study participant required oxygen supplement on admission, and the median $\mathrm{SpO}_{2}$ was $98 \%$ (97-99\%). The mean NEWS2 score was $0.59 \pm 0.7$. All patients were managed in negatively pressured and double-doored cubicles within isolation wards. Six patients received a combination of interferon beta-1b, lopinavir-ritonavir and ribavirin; 9 patients received lopinavir-ritonavir and ribavirin; 13 patients received hydroxychloroquine and interferon beta-1b; and 6 patients received supportive care only. No patients received corticosteroid in the study.

Physiology parameters monitoring. During the median hospital stay of 15 days (9-20 days), a total of 1231 manual measurement of physiology parameters were made for these 34 patients, which consumed 747 sets of PPE. As expected, the time-stamped physiology parameters including heart rate and oxygen saturation captured using wearable biosensors correlated with those from manual measurement with Pearson correlation coefficients (r) of 0.96 (95\% CI 0.95-0.97), and 0.87 (95\% CI 0.81-0.91) respectively (Appendix Figure 1). 
Correlation between Biovitals Index, NEWS2 and viral load. The 24-h average Biovitals Index of individual patients was positively associated with the 24-h NEWS2. (Fig. 2A) The 24-h NEWS2 increased progressively with increasing 24 -h average Biovitals Index from $0.6 \pm 0.4$ with the Biovitals Index between 0.0 and 0.2 , to $4.1 \pm 1.6$ with the Biovitals Index between 0.7 and $1.0(p<0.0001)$. To our surprise, the increase in viral load from respiratory samples as determined with RT-PCR Ct value for SARS-CoV-2 was associated with increasing 24-h average Biovitals Index $(p<0.0001)$, but not with the 24-h NEWS2 $(p=0.004, r=0.15)$. (Fig. 2B,C). Furthermore, the diagnostic performance to identify moderate/high viral load in the respiratory samples was compared between the 24-h average Biovital Index and the 24-h NEWS2. The area under the curve of 24-h average Biovital Index to identify moderate/high viral load was 0.87 (95\% CI 0.83-0.90), significantly larger than that of the 24 -h NEWS2 $(0.68,95 \%$ CI $0.65-0.71)$. Specifically, the 24 -h average Biovitals Index $>0.5$ correctly identified $100 \%$ moderate/high viral load with a false positive rate of $0.0 \%$ and a false negative rate of $11.9 \%$. On the other hand, the 24 -h NEWS2 $\geq 5$ identified $80 \%$ moderate/high viral load with a false positive rate of $0.4 \%$ and a false negative rate of $88.6 \%$ (Fig. 2D).

Prediction of clinical worsening events. A total of 17 clinical worsening events occurred in these 34 COVID-19 patients during hospitalization. Biovitals Index alerts detected 16 out of 17 events (94.1\%) prior to the clinical events getting noticed in ward with prediction time interval of $21.0 \mathrm{~h}$, ranging from 6 to $39 \mathrm{~h}$. On the other hand, there were 2 Biovitals Index alerts without actual clinical worsening event within the subsequent 3 days (false positive). The area under the curve was 0.93 (95\% CI: 0.89-0.95) with the optimal cut-off at sensitivity and specificity of $94.1 \%$ and $88.9 \%$ respectively. The performance of Biovitals Index to predict clinical worsening events was then compared with the 24-h NEWS2. The area under the curve for 24-h NEWS2 to predict clinical worsening event was only 0.64 (95\% CI 0.61-0.67) with the optimal cut-off at sensitivity and specificity of $29.4 \%$ and $85.7 \%$ respectively (Fig. $2 \mathrm{E}, \mathrm{F}$ ).

Prediction of length of stay. The Biovitals Index progressively decreased as patients improved. To estimate the remaining length of stay, machine learning-based projection of the Biovitals Index reduction trend was performed. After collecting sufficient data from each patient for fitting into the machine learning model over a variable number of days, one-time predictions of the remaining length of stay were made (Supplementary Appendix and Appendix Figure 2). The median time spent for generating the prediction was 8 days (3-10 days). For COVID-19 patients with an actual total hospital stay $\leq 15$ days, there were no statistically significant difference between the actual length of stay and the predicted length of stay. However, for those with actual hospital stay $>15$ days, the predicted length of stay was significantly shorter than the actual hospital stay (17.8 days (1425 days) vs. 21.5 days (16-31 days), $p=0.031$ ) (Fig. 2G). Nonetheless, compared with NEWS2 score on admission to predict prolonged hospital stay ( $>10$ days), the area under the curve of the machine learning model of 0.78 (95\% CI $0.72-0.82)$ with the optimal cut-off at sensitivity and specificity of $66.7 \%$ and $72.7 \%$, was superior to the NEWS2 with area under the curve of 0.58 (95\% CI $0.53-0.64)$ with the optimal cut-off at sensitivity and specificity of $51.7 \%$ and $59.1 \%$.

\section{Discussion}

In this study we demonstrated feasibility and potential clinical application of an wearable biosensor and machine learning-based remote monitoring platform for managing hospitalized COVID-19 patients. We first showed strong correlation between physiology parameters including heart rate and oxygen saturation obtained from the wearable biosensors and manual measurements. Furthermore, a machine learning-derived health index, Biovitals Index, generated using machine learning-based analysis of multivariate longitudinal physiology parameters demonstrated a superior ability to discriminate COVID-19 patients with moderate/high viral load from those with undetectable or low viral load, to recognize clinical worsening events early, and to predict hospital discharge, than the widely adopted NEWS2.

Prior studies have demonstrated that continuous monitoring is superior to intermittent monitoring in detecting deterioration in hospitalized patients ${ }^{31-34}$. However, continuous monitoring outside the intensive care unit remains uncommon. Setting up continuous monitoring systems used to be cumbersome as it involves connecting patients to sensor devices with numerous electrodes and cables, which restrict patient activities to the bed area. Also, data transmission were highly reliant on in-house telecommunication infrastructure. In contrast, wearable device such as armband or wristband incorporates multiple biosensors in a single form-factor, which allows a higher degree of patient mobility without the constraints of physical wirings. More importantly, data transmission through cellular network avoids the need of installing additional in-house telecommunication infrastructure, allows rapid deployment, and provides versatile and scalable solutions to suit different clinical applications such as emergent response to the COVID-19 pandemic. In this study, the platform covering 4 isolation wards and 2 stepdown wards located in 2 different hospital buildings was successfully deployed within $24 \mathrm{~h}$. Amidst the pandemic, fewer manual measurement of physiology parameters not only lessens the workload of healthcare workers, but also reduces their exposure to the virus, risk of in-hospital infection and consumption of PPE.

One challenge of continuous monitoring of physiology parameters was to determine when to alert clinicians when alarming signs emerge. Conventional remote monitoring systems alert clinicians when any of the monitored physiology parameters exceed pre-determined thresholds and often overload clinicians with false alarms. Early warning systems such as NEWS2 allow more precise deterioration prediction by concurrently considering multiple physiology parameters. In our study, Biovitals Index generated by autonomous analysis of the continuous streams of physiology data predicted clinical deterioration with superior accuracy than the widely adopted early warning system NEWS2, in additional to identifying patients with high viral load. The superior accuracy is partly attributed to the massive amount of physiology parameters available for analysis, which provided a 
A

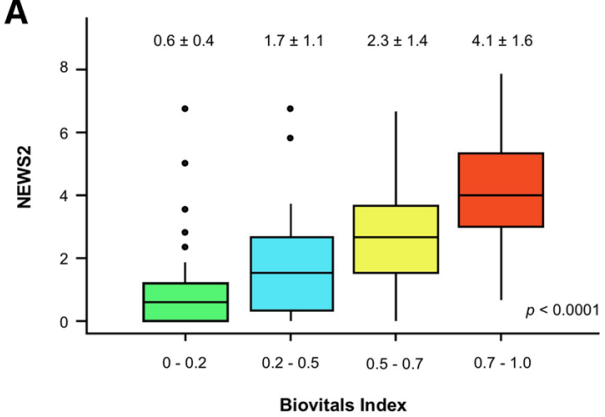

C

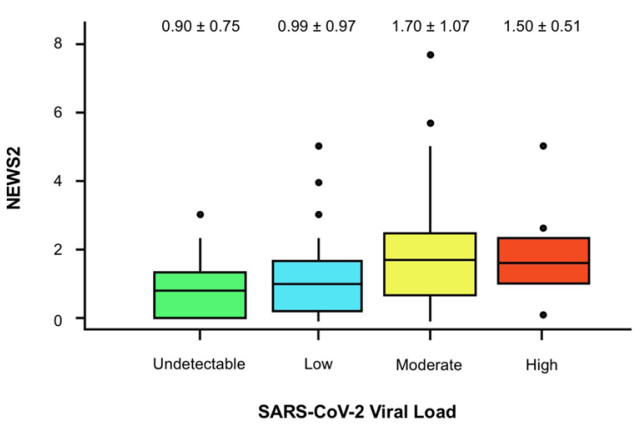

E

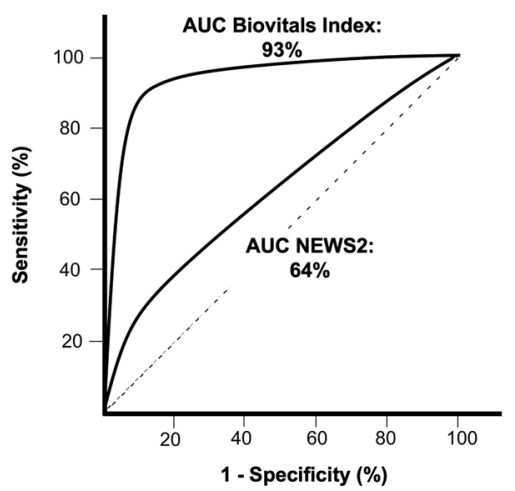

G

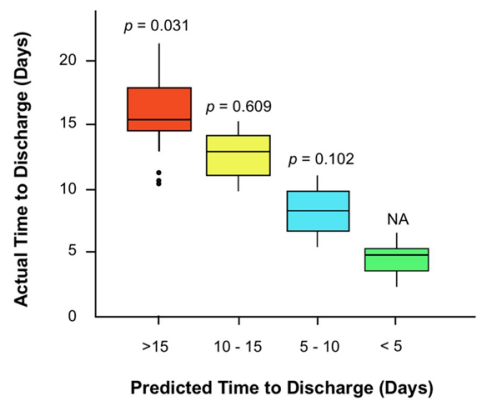

B

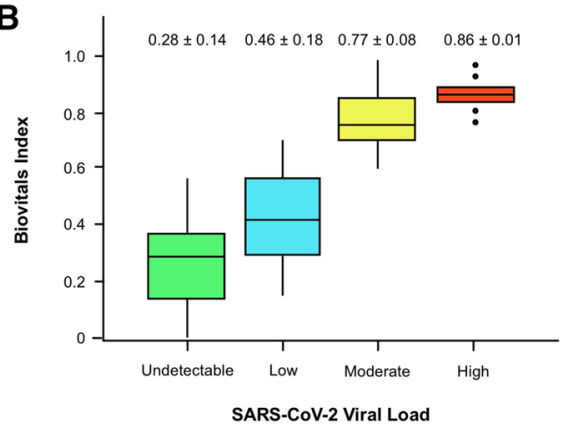

D Moderate-to-High SARS-CoV-2 Viral Load

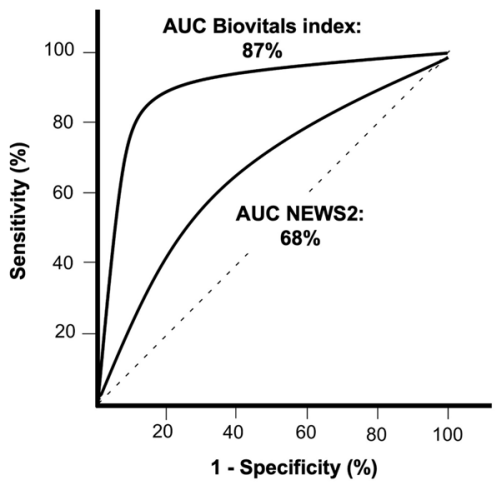

$\mathbf{F}$

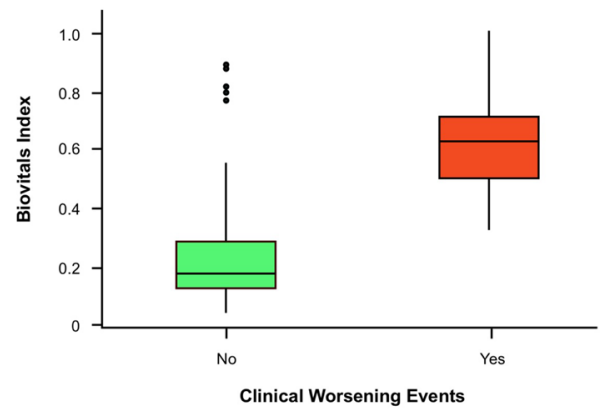

Figure 2. (A) 24-h average Biovitals Index positively correlated with 24-h NEWS2. (B,C) Respiratory samples SARS-CoV-2 viral load determined by RT-PCR cycle threshold (Ct) values correlated with 24 -h average Biovitals Index $(p<0.0001)$ but did not correlate with 24-h NEWS2 $(p=0.004, r=0.15)$. (D) The area under the curve of 24-h average Biovital Index to identify moderate/high viral load was 0.87 (95\% CI 0.83-0.90). (E,F) Biovitals Index predicted clinical worsening events with the area under the curve of 0.93 ( $95 \% \mathrm{CI} 0.89-0.95$ ) with the optimal cut-off at sensitivity and specificity of $94.1 \%$ and $88.9 \%$ respectively. 24 -h NEWS2 predicted clinical worsening events with area under the curve of 0.64 (95\% CI 0.61-0.67) with the optimal cut-off at sensitivity and specificity of $29.4 \%$ and $85.7 \%$ respectively. (G) Actual time-to-discharge in relation to the predicted time-to-discharge derived from machine learning-based projection. 
more comprehensive representation of each patient's health status than intermittent manual measurements. Furthermore, the current system also took historical physiology data of each patient into account and multiple machine learning techniques were used to allow accurate analysis, such data pre-processing, data segmentation, feature extraction, anomaly detection and semi-supervised multivariate regression. Most of the aforementioned elements were not possible with early warning systems like NEWS2. Moving forward, it is possible that machine learning-based analysis of multivariate longitudinal physiology parameters will be increasing utilized in other clinical settings as well. The improved precision in predicting clinical deterioration allows more prompt and targeted intervention to be delivered.

To further validate the value of this wearable biosensor and machine learning-based remote monitoring platform in clinical practice, it is necessary to conduct adequately powered randomized controlled trials to demonstrate its effectiveness in improving patient outcome. In addition to having accurate machine learningbased predictions and alerts, it is also important to appropriately define intervention for each clinical alert. In the setting of COVID-19, as to date since there is still a lack of effective therapeutics, clinical alerts will mostly allow accurate triage of patients and efficient allocation of hospital resources.

Limitations. This study had several limitations. First, it was limited by the relatively small sample size and a single-centred observational design. Second, the study included patients with mild or moderate COVID-19 on admission, limiting the generalizability of the patients with severe or critical disease. Nonetheless, patients with severe or critical COVID-19 are most likely triaged for high dependency units or intensive care units are not the target candidates for remote monitoring. Last but not least, while technically attractive, potential privacy concerns may arise with sensitive physiological data from individual patients being transferred and stored in the networks, necessitating appropriate regulatory measures for proper privacy protect prior to large-scale implementationn ${ }^{35}$.

\section{Conclusion}

Wearable biosensors coupled with machine learning-based multivariate analysis of physiology parameters allowed accurate detection of COVID-19 patients at risk of deterioration.

\section{Data sharing statement}

Deidentified clinical and post-processing physiology data will be available from the corresponding author on reasonable request after obtaining approval by the investigators and signing data access agreement, from date of publication to one year after publication.

Received: 26 August 2020; Accepted: 21 January 2021

Published online: 23 February 2021

\section{References}

1. Chu, H. et al. Comparative replication and immune activation profiles of SARS-CoV-2 and SARS-CoV in human lungs: An ex vivo study with implications for the pathogenesis of COVID-19. Clin. Infect. Dis. https://doi.org/10.1093/cid/ciaa410 (2020).

2. Chu, H. et al. Comparative tropism, replication kinetics, and cell damage profiling of SARS-CoV-2 and SARS-CoV with implications for clinical manifestations, transmissibility, and laboratory studies of COVID-19: an observational study. Lancet Microbe. 1, e14-e23 (2020).

3. Guan, W. J. et al. Clinical characteristics of coronavirus disease 2019 in China. N. Engl. J. Med. https://doi.org/10.1056/NEJMo a2002032 (2020).

4. Chan, J. F. et al. A familial cluster of pneumonia associated with the 2019 novel coronavirus indicating person-to-person transmission: A study of a family cluster. Lancet 395(10223), 514-523. https://doi.org/10.1016/S0140-6736(20)30154-9 (2020).

5. Shoukat, A. et al. Projecting demand for critical care beds during COVID-19 outbreaks in Canada. CMAJ https://doi.org/10.1503/ cmaj.200457 (2020).

6. Li, R. et al. Estimated demand for US hospital inpatient and intensive care unit beds for patients with COVID-19 based on comparisons with Wuhan and Guangzhou, China. JAMA Netw. Open 3(5), e208297. https://doi.org/10.1001/jamanetworkopen $.2020 .8297(2020)$.

7. Litton, E. et al. Surge capacity of intensive care units in case of acute increase in demand caused by COVID-19 in Australia. Med. J. Aust. 212(10), 463-467. https://doi.org/10.5694/mja2.50596 (2020).

8. Gilbert, M. et al. Preparedness and vulnerability of African countries against importations of COVID-19: A modelling study. Lancet 395(10227), 871-877. https://doi.org/10.1016/S0140-6736(20)30411-6 (2020).

9. Stang, A., Stang, M. \& Jockel, K. H. Estimated use of intensive care beds due to COVID-19 in Germany over time. Dtsch Arztebl Int. 117(19), 329-335. https://doi.org/10.3238/arztebl.2020.0329 (2020).

10. Zhou, F. et al. Clinical course and risk factors for mortality of adult inpatients with COVID-19 in Wuhan, China: A retrospective cohort study. Lancet 395(10229), 1054-1062. https://doi.org/10.1016/S0140-6736(20)30566-3 (2020).

11. Wu, C. et al. Risk factors associated with acute respiratory distress syndrome and death in patients with coronavirus disease 2019 pneumonia in Wuhan, China. JAMA Intern. Med. https://doi.org/10.1001/jamainternmed.2020.0994 (2020).

12. Smith, G. B. et al. The ability of the National Early Warning Score (NEWS) to discriminate patients at risk of early cardiac arrest, unanticipated intensive care unit admission, and death. Resuscitation 84(4), 465-470. https://doi.org/10.1016/j.resuscitat ion.2012.12.016 (2013).

13. Pimentel, M. A. F. et al. A comparison of the ability of the National Early Warning Score and the National Early Warning Score 2 to identify patients at risk of in-hospital mortality: A multi-centre database study. Resuscitation 134, 147-156. https://doi. org/10.1016/j.resuscitation.2018.09.026 (2019).

14. Faisal, M. et al. Computer-aided National Early Warning Score to predict the risk of sepsis following emergency medical admission to hospital: A model development and external validation study. CMAJ 191(14), E382-E389. https://doi.org/10.1503/cmaj.18141 8 (2019).

15. Ranney, M. L., Griffeth, V. \& Jha, A. K. Critical supply shortages-the need for ventilators and personal protective equipment during the Covid-19 pandemic. N. Engl. J. Med. 382(18), e41. https://doi.org/10.1056/NEJMp2006141 (2020). 
16. Kamerow, D. Covid-19: The crisis of personal protective equipment in the US. BMJ 369, m1367. https://doi.org/10.1136/bmj. m1367 (2020).

17. Coronavirus disease 2019 (COVID-19) Situation Report-83 (2020). https://www.whoint/docs/default-source/coronaviruse/situa tion-reports/20200411-sitrep-82-covid-19pdf?sfvrsn=74a5d15_2. Accessed 26 May 2020.

18. van Vliet, M. et al. Continuous non-invasive monitoring of the skin temperature of HSCT recipients. Support Care Cancer 18(1), 37-42. https://doi.org/10.1007/s00520-009-0627-1 (2010).

19. Pevnick, J. M. et al. Wearable technology for cardiology: An update and framework for the future. Trends Cardiovasc. Med. 28(2), 144-150. https://doi.org/10.1016/j.tcm.2017.08.003 (2018).

20. Buekers, J. et al. Wearable finger pulse oximetry for continuous oxygen saturation measurements during daily home routines of patients with chronic obstructive pulmonary disease (COPD) over one week: Observational study. JMIR Mhealth Uhealth 7(6), e12866. https://doi.org/10.2196/12866 (2019).

21. Georgiou, K. et al. Can wearable devices accurately measure heart rate variability? A systematic review. Folia Med. Plovdiv. 60(1), 7-20. https://doi.org/10.2478/folmed-2018-0012 (2018).

22. Chu, M. et al. Respiration rate and volume measurements using wearable strain sensors. NPJ Digit. Med. 2, 8. https://doi. org/10.1038/s41746-019-0083-3 (2019).

23. Aliverti, A. Wearable technology: Role in respiratory health and disease. Breathe (Sheff) 13(2), e27-e36. https://doi. org/10.1183/20734735.008417 (2017).

24. Majumder, S., Mondal, T. \& Deen, M. J. Wearable sensors for remote health monitoring. Sensors (Basel) https://doi.org/10.3390/ s17010130 (2017).

25. Chan, A. W. et al. SPIRIT 2013 statement: Defining standard protocol items for clinical trials. Ann. Intern. Med. 158(3), 200-207. https://doi.org/10.7326/0003-4819-158-3-201302050-00583 (2013).

26. Chan, A. W. et al. SPIRIT 2013 explanation and elaboration: Guidance for protocols of clinical trials. BMJ 346, e7586. https://doi. org/10.1136/bmj.e7586 (2013).

27. Moher, D. et al. The CONSORT statement: Revised recommendations for improving the quality of reports of parallel group randomized trials. BMC Med. Res. Methodol. 1, 2. https://doi.org/10.1186/1471-2288-1-2 (2001).

28. Altman, D. G. et al. The revised CONSORT statement for reporting randomized trials: Explanation and elaboration. Ann. Intern. Med. 134(8), 663-694. https://doi.org/10.7326/0003-4819-134-8-200104170-00012 (2001).

29. Chen, J. et al. Biovitals: A personalized multivariate physiology analytics using continuous mobile biosensors. Conf. Proc. IEEE Eng. Med. Biol. Soc. 2019, 3243-3248. https://doi.org/10.1109/EMBC.2019.8856482 (2019).

30. National Early Warning Score (NEWS) 2. Standardising the assessment of acute-illness severity in the NHS Updated report of a working party. Executive summary and recommendations. (The Royal College of Physicians, London, 2017).

31. Sulter, G. et al. Admitting acute ischemic stroke patients to a stroke care monitoring unit versus a conventional stroke unit: A randomized pilot study. Stroke 34(1), 101-104. https://doi.org/10.1161/01.str.0000048148.09143.6c (2003).

32. Cavallini, A. et al. Role of monitoring in management of acute ischemic stroke patients. Stroke 34(11), 2599-2603. https://doi. org/10.1161/01.STR.0000094423.34841.BB (2003).

33. Langhorne, P. et al. Very early rehabilitation or intensive telemetry after stroke: A pilot randomised trial. Cerebrovasc. Dis. 29(4), 352-360. https://doi.org/10.1159/000278931 (2010).

34. Varela, M. et al. "Catching the spike and tracking the flow": Holter-temperature monitoring in patients admitted in a general internal medicine ward. Int. J. Clin. Pract. 65(12), 1283-1288. https://doi.org/10.1111/j.1742-1241.2011.02794.x (2011).

35. Gerke, S. et al. Regulatory, safety, and privacy concerns of home monitoring technologies during COVID-19. Nat. Med. 26, 1176-1182 (2020).

\section{Acknowledgements}

Everion wearable biosensors were donated by Biofourmis Singapore Pte. Ltd and Harmony Medical Inc. Smartphones and cellular data were supported by PCCW Ltd.

\section{Author contributions}

K.C.U., C.K.W., Y.M.L., R.C.F.T., M.D.M., R.K.S., I.F.N.H. and C.W.S. contributed to the conception and design of the study. K.C.U., C.K.W., Y.M.L., J.C.Y.L., F.C.C.T., W.H.L., Y.M.L., S.M.L., A.C., R.C.F.T., I.F.N.H. and C.W.S. contributed to the acquisition of data. Data analysis and interpretation will be conducted by H.C., S.W., X.Z., M.Y., E.W., S.C.C., R.K.S. and C.W.S. K.C.U., C.K.W., H.C., and C.W.S. wrote first draft of the protocol. R.K.S. and I.F.N.H. revised the manuscript critically for important intellectual content. All authors have read and approved the final version of the manuscript to be published.

\section{Competing interests}

HC, SW, XZ, MY, EW, SCC, MDM and RKS are employed by Biofourmis Singapore Pte. Ltd, which donated Everion wearable biosensors. SML, AC and RCFT are employed by Harmony Medical Inc., which donated Everion wearable biosensors.

\section{Additional information}

Supplementary Information The online version contains supplementary material available at https://doi. org/10.1038/s41598-021-82771-7.

Correspondence and requests for materials should be addressed to C.-W.S.

Reprints and permissions information is available at www.nature.com/reprints.

Publisher's note Springer Nature remains neutral with regard to jurisdictional claims in published maps and institutional affiliations. 
(c) (i) Open Access This article is licensed under a Creative Commons Attribution 4.0 International cc) License, which permits use, sharing, adaptation, distribution and reproduction in any medium or format, as long as you give appropriate credit to the original author(s) and the source, provide a link to the Creative Commons licence, and indicate if changes were made. The images or other third party material in this article are included in the article's Creative Commons licence, unless indicated otherwise in a credit line to the material. If material is not included in the article's Creative Commons licence and your intended use is not permitted by statutory regulation or exceeds the permitted use, you will need to obtain permission directly from the copyright holder. To view a copy of this licence, visit http://creativecommons.org/licenses/by/4.0/.

(C) The Author(s) 2021 